\title{
Mujeres en STEM, un reto educativo
}

\author{
Women in STEM, an educational challenge
}

\author{
SANDOVAL-PALOMARES, Jessica †*, GARCÍA-CERVANTES, Heraclio, BLANCO-MIRANDA, \\ Alan David y CARRILLO-HERNÁNDEZ, Didia
}

Universidad Tecnológica de León, Cuerpo Académico: Bioingeniería, México.

ID $1^{\text {er }}$ Autor: Jessica, Sandoval-Palomares / ORC ID: 0000-0002-3294-0916, Researcher ID Thomson: S-9841-2018, CVU CONACYT ID: 827848

ID $1^{\mathrm{er}}$ Coautor: Heraclio, García-Cervantes / ORC ID: 0000-0002-4229-9229, Researcher ID Thomson: X-5622-2019, CVU CONACYT ID: 290829

ID $2^{\text {do }}$ Coautor: Alan David, Blanco-Miranda / ORC ID: 0000-0002-8595-8634, Researcher ID Thomson: W-9701-2019, CVU CONACYT ID: 298274

ID3 ${ }^{\text {er }}$ Coautor: Didia, Carillo-Hernández / ORC ID: 0000-0001-9989-5884, Researcher ID Thomson: ABF-4839-2020, CVU CONACYT ID: 936937

DOI: $10.35429 / J O C S .2020 .23 .7 .20 .29$

Recibido: 20 de Julio, 2020; Aceptado 30 de Diciembre, 2020

\begin{abstract}
Resumen
La ciencia, la tecnología y la innovación son elementos para dar respuesta a los desafíos que es necesario afrontar, como son, entre otros, el cambio climático, las energías renovables, la alimentación de la humanidad, la salud y la administración de los recursos. En la actualidad, las mujeres tienen un bajo porcentaje de representación en carreras de Ciencia, Tecnología, Ingeniería y Matemática, STEM por sus siglas en inglés; la brecha de genero subsiste en el tema de lo laboral, donde se requiere que las empresas permitan el ingreso de la mujer en posiciones de liderazgo. La ONU, para dar respuesta a esta evidente disparidad, en 2015 establece un día internacional para reconocer el importante papel que tiene en la ciencia y la tecnología, mismo que es proclamado el 11 de febrero como Día Internacional de la Mujer y la Niña en la Ciencia. La presente investigación analiza la percepción de mujeres que se formaron en carreras STEM, con el propósito de conocer su percepción en seis aspectos, a saber; percepción sobre su formación académica, habilidad para aprender y resolver problemas en áreas STEM, apoyo social, docente o familiar, formación académica, satisfacción en su trabajo y clima laboral, estereotipos de género y análisis de las habilidades o competencias requeridas.
\end{abstract}

STEM, Científicas, Brecha de género, Competencias

\begin{abstract}
Science, technology and innovation are elements to respond to the challenges that must be faced, such as, among others, climate change, renewable energies, the nutrition of humanity, health and the administration of resources. Currently, women have a low percentage of representation in science, technology, engineering and mathematics majors, STEM, for its acronym in English; the gender gap persists in the labor issue, where companies are required to allow women to enter leadership positions. The ONU, to respond to this evident disparity, in 2015 establishes an international day to recognize the important role it has in science and technology, which is proclaimed on February 11 as International Day of Women and Girls in the Science. This research analyzes the perception of women who were trained in STEM careers, with the purpose of knowing their perception in six aspects, namely; Perception of their academic training, ability to learn and solve problems in STEM areas, social, educational or family support, academic training, satisfaction in their work and the work environment, gender stereotypes and the analysis of the skills or competencies required.
\end{abstract}

STEM, Scientific, Gender gap, Skills

Citación: SANDOVAL-PALOMARES, Jessica, GARCÍA-CERVANTES, Heraclio, BLANCO-MIRANDA, Alan David y CARRILLO-HERNÁNDEZ, Didia. Mujeres en STEM, un reto educativo. Revista de Sociología Contemporánea. 2020. 723:20-29.

\footnotetext{
* Correspondencia del Autor (Correo electrónico: jspalomares@utleon.edu.mx)

$\dagger$ Investigador contribuyendo como primer autor.
} 


\section{Introducción}

El acrónimo STEM se refiere al conjunto de disciplinas relacionadas con la ciencia, la tecnología, la ingeniería y las matemáticas, la educación STEM forma a profesionales que liderean los cambios tecnológicos; sin embargo, son escasos cuando se comparan con otros ámbitos como son las de humanidades, económico administrativa, salud, entre otras.

Requiere atención especial la inclusión de las mujeres en estas disciplinas, la cual es inferior a la participación de los hombres, en la revisión de la literatura se identifica que únicamente un $35 \%$ de estudiantes en áreas STEM a nivel mundial son de género femenino, traduciéndose en una escasa representación en los empleos ofertados, agrandando la brecha de género (Arredondo, Vázquez y Vázquez, 2019). Para solventar la situación y crear un marco adecuado para que las mujeres accedan a las STEM y a los empleos ofertados, es necesario dar importancia a la orientación vocacional y profesional temprana para reducir la brecha entre hombres y mujeres en cuestión de igualdad de oportunidades.

Una primera interpretación pone especial énfasis en el rechazo que muchas niñas tienen hacia las materias relacionadas con STEM, lo cual está condicionado por la percepción de dificultad y la actitud hacia el fracaso, los bajos resultados académicos comparativos con otras asignaturas, el esfuerzo para afrontar los exámenes, la comparación con los niños al conocer los resultados e incluso cierto rechazo cultural de las familias hacia estas disciplinas.

En estudios realizados por la Organización para la Cooperación y el Desarrollo Económicos (OCDE), se identifica que las niñas obtienen solo una diferencia de menos de 5 puntos con respecto a los niños en matemáticas (PISA, 2018), y ellos solo obtuvieron mejores resultados que ellas en menos de la mitad de los 79 países participantes en el mencionado estudio. En ciencias, las chicas obtuvieron mejores resultados que los chicos en 2 puntos porcentuales y en alrededor de la mitad de los países que se analizaron; con estos resultados se observa que la brecha de género en ciencias no es estadísticamente significativa. Para solventar el problema, se requiere trabajo en el aula por parte de los docentes, además de ofrecer más y mejor información a las familias.
De acuerdo con los resultados de PISA, se observa que la brecha de género se empieza a agrandar en la educación secundaria, para posteriormente trasladarse al ámbito laboral.

En la presente investigación se analizarán diversas habilidades, estrategias, recursos internos, apoyos sociales y talento de mujeres profesionistas de áreas STEM, con la finalidad de proporcionar información de factores que las llevaron a culminar con éxito su formación escolar, y que en dado momento, pueda servir como un elemento base para el fortalecimiento de los modelos de aprendizaje en niñas y jóvenes.

\section{Justificación}

A nivel nacional y según cifras de la OCDE, en 2016 sólo el 17\% de las personas entre 25 y 64 años, contaban con estudios de educación superior, porcentaje muy por abajo en relación con el que se obtiene en los países miembros de la Organización para la Cooperación y el Desarrollo Económico (OCDE, Panorama de la Educación 2017); en el mismo informe, las mujeres representan el 30\% de estudiantes de nuevo ingreso en educación superior a las áreas STEM.

En la revisión de la literatura, se encontró que hay más cantidad de hombres que de mujeres en carreras científicas, tecnológicas, ingenierías y matemáticas (Bystydzienski y Bird, 2006; Shapiro y Sax, 2011, citado por Inga y Tristán, 2020). En México, de acuerdo con datos referidos por la UNESCO, únicamente el $35 \%$ de los investigadores adscritos al Sistema Nacional de Investigadores (SNI) son mujeres, y solo el $24.5 \%$ de los miembros de la Academia Mexicana de Ciencias son de género femenino (Jiménez, 2017).

Las razones de esta menor participación de las mujeres son variadas, Morales y Morales (2020) proporcionan una clasificación que permite explicar esta diferencia:

a. Explicación psicológica: Se enfoca en el individuo, así como algunos procesos cognitivos como el autoconcepto, creencias, percepciones y diferencias en intereses.

b. Explicación sociocultural: Destaca la importancia de la cultura, la influencia social y familiar, estereotipos de género.

SANDOVAL-PALOMARES, Jessica, GARCÍACERVANTES, Heraclio, BLANCO-MIRANDA, Alan David y CARRILLO-HERNÁNDEZ, Didia. Mujeres en STEM, un reto educativo. Revista de Sociología Contemporánea. 2020 
c. Explicación biológica: Proporciona las diferencias del sexo anatómico y de género.

\section{Revisión de la literatura}

El Programme for International Student Assessment (PISA), evalúa los conocimientos y habilidades de estudiantes que están cercanos a finalizar su educación, y los cuales son necesarios para su participación en la sociedad del saber. La evaluación muestra a aquellos países que han alcanzado un buen rendimiento y aprovechamiento del aprendizaje, al mismo tiempo, identifica el reparto equitativo de las oportunidades en los distintas áreas del saber.

Las pruebas de PISA se aplican cada tres años, examinando el aprovechamiento de jóvenes de 15 años en áreas temáticas clave (lectura, matemáticas y ciencias), también analizan una gama amplia de resultados educativos, entre ellos: la motivación de los alumnos por aprender, la concepción que éstos tienen sobre sí mismos y sus estrategias de aprendizaje. Se ha identificado que los niños obtienen mejores puntuaciones que las niñas en más de la mitad de los países que pertenecen a la OCDE, sin embargo, esta diferencia no es significativa en el caso de menores superdotados. El hecho de que se muestren diferencias en el desempeño de niñas y niños, y que varíen con respecto al nivel socioeconómico o en menores con aptitudes sobresalientes, mostraron la necesidad de incluir variables de corte psicológico, sociocultural y biológico en el instrumento de medición.

Desde el ámbito biológico, se ha identificado que niños y niñas de entre 12 y 13 años, reportan diferencias en la forma de cómo abordan las pruebas matemáticas, mientras que los varones obtienen mayor calificación en los ítems de resolución de problemas, las mujeres en cambio lo hacen en los ejercicios mentales de aritmética (Goetz, et al., 2008, citado por Canché, Farfán y Simón, 2011). Sin embargo, aún no se encuentran evidencias neurocientíficas contundentes de una diferencia de habilidades matemáticas entre hombres y mujeres. Desde la revisión cognitiva, en específico en el tema de autoconcepto y creencias de las propias habilidades matemáticas, se encontró que los varones tienen una mayor confianza en sus habilidades que las niñas (Goetz, 2008, citado por Canché, Farfán y Simón, 2011).
En el aspecto sociocultural, se ha encontrado que el nivel socioeconómico incide en los estereotipos de género con relación a las matemáticas, y tiene un impacto en la elección de carrera (Del Río, Strasser y Susperreguy, 2016). Desde lo psicosocial, Del Río, Strasser y Susperreguy (2016), analizaron a menores en edad preescolar, a sus profesores y padres de familia; encontraron estereotipos de género en niños y niñas en edad preescolar, manifestándose en mensajes recibidos por sus cuidadores y la influencia que ellos tienen en la formación de las creencias desde edades tempranas.

\section{Método}

Para fines de la presente investigación se selecionó el diseño de tipo exploratoro, al ser son estudios que buscan una visión general acerca de una determinada realidad, de un tema que ha sido poco estudiado.

El instrumento de medición seleccionado fue una encuesta con 19 ítems, resueltas a través de una escala tipo Likert, donde 1 es totalmente en desacuerdo y 5 es totalmente de acuerdo. En referencia al análisis e interpretación de resultados, el instrumento fue resuelto en forma electrónica, vía formulario electrónico, ala muestra seleccionada fueron 64 mujeres profesionistas en áreas STEM.

\section{Resultados}

Se recibieron un total de 64 instrumentos resueltos, distribuidos de la siguiente forma: las profesionistas manifestaron trabajar en los siguientes lugares: universidades públicas (25), centros de investigación (14), empresas (10), independientes (15); manifestaron residir en las siguientes ciudades: Región Bajío (25), Monterrey (5), Guadalajara (8), CDMX (13), Boston (3), Arizona (4), Ontario, (6); todas las encuestadas son mexicanas de nacimiento $y$ realizaron sus estudios en el territorio nacional. 


\section{Datos demográficos}

El $84.37 \%$ de las profesionistas cuentan con posgrado: $59.37 \%$ maestría, $21.87 \%$ doctorado, $3.12 \%$ postdoctorado; y solo el $15.63 \%$ cuenta únicamente con estudios de licenciatura; sus áreas de conocimiento se encuentran distribuidas de la siguiente forma: Área VII: Ingenierías con el 56.25\%, Área I: Físico-Matemáticas 25\%, área II: Biología y Química con 9\%, y finalmente, Área III: Ciencias Médicas y de la salud con $3 \%$.

Las carreras base, agrupadas por área, se mencionan a continuación:

- Ingenierías: Ingeniería en sistemas (industrial y computacionales), desarrollo e innovación, en mecánica de control y automatización.

- Físico-matemático: Ingeniería en ciencias de la computación, Licenciatura en actuaría, estadística y matemáticas.

Biología y química: Ingeniería en ciencias químicas e Ingeniería en tecnología de polímeros.

- Ciencias médicas y de la salud: Ingeniería en ciencias biomédicas, Licenciatura en cirujano dentista y en médico cirujano; Ingeniería química bacteriológica y en tecnología de alimentos.

En referencia al aspecto laboral, se reportan los siguientes datos:

- En el ámbito académico: docentes en universidad pública (nacional y en extranjero), secretaría de vinculación.

- Ámbito de centros tecnológicos: directoras académicas, administradoras de proyectos, e investigadoras.

- Sector industrial o empresarial: control de proyectos en ámbito nacional y extranjero, cadena de suministro, analista, y estadista.

- Emprendedoras o profesionistas independientes: en áreas de capacitación y área de servicios.
El rango de edad donde se encuentran corresponde de 38 a 49 años, con $58.3 \%$ del total, con lo que se infiere que cuentan con una importante experiencia profesional.

Para fines de análisis, las preguntas se clasifican en cinco bloques:

a. Percepción sobre la formación académica;

b. Habilidad para aprender y resolver problemas en áreas STEM;

c. Apoyo social, docente o familiar;

d. Formación académica, satisfacción en su trabajo y el clima laboral;

e. Estereotipos de género;

f. Análisis de las habilidades o competencias.

Se presentan a continuación los resultados del instrumento:

\section{a. Percepción sobre la formación académica: el bloque consta de 3 ítems.}

En la pregunta: ¿considero que durante mi educación primaria recibí una formación sólida en el área de matemáticas? Se identifica que 32 mujeres, que corresponde a un $50 \%$, mencionan estar totalmente de acuerdo con la formación que recibieron. La gráfica se presenta a continuación:

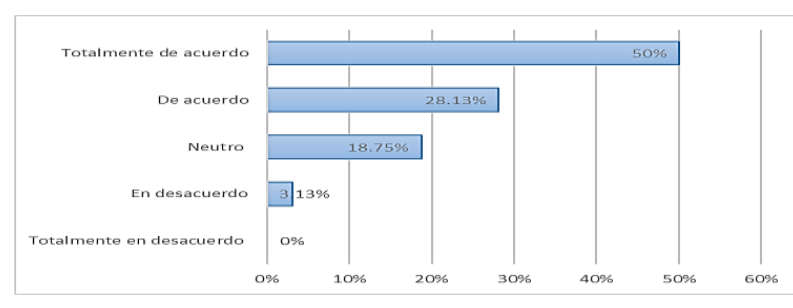

Gráfico 1 Formación académica

Fuente: Elaboración propia

Las respuestas para la afirmación: "Considero que el estudio de algunas materias como son matemáticas, Biología, Química, Geografía y Ciencias, me han ayudado a desarrollar habilidades de resolución de problemas", indican que el $87.5 \%$, (56 personas), informan estar totalmente de acuerdo, el $9.37 \%$ de acuerdo y el $3.12 \%$ dan una respuesta neutral. La gráfica se presenta a continuación: 


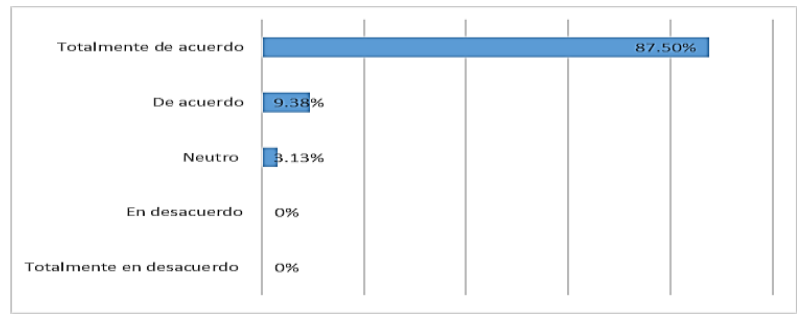

Gráfico 2 Desarrollo de habilidades

Fuente: Elaboración propia

Las respuestas para la afirmación: "Considero que durante mi formación académica tuve excelentes docentes de asignaturas STEM", muestran una distribución interesante: el $42.18 \% \%$ indica estar totalmente de acuerdo y $35.93 \%$ de acuerdo, el restante de las respuestas, se distribuyen en neutral $14.06 \%$, en desacuerdo $4.68 \%$ y totalmente en desacuerdo con $3.12 \%$. La gráfica se presenta a continuación:

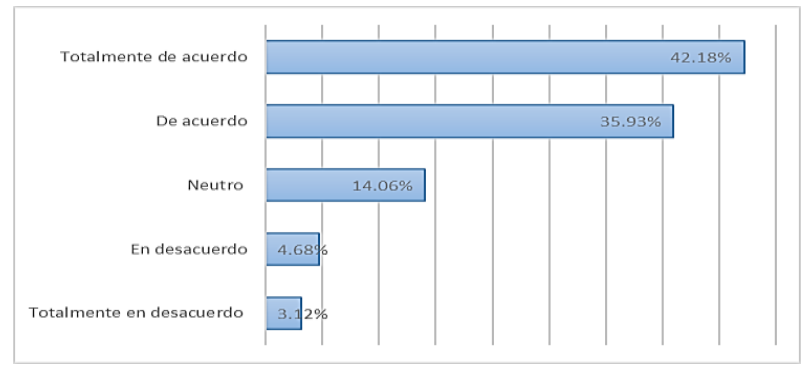

Gráfico 3 Formación STEM

Fuente: Elaboración propia

b. $\quad$ El segundo bloque, mide la habilidad para aprender y resolver problemas en áreas STEM, y su influencia en el desarrollo académico; consta de 2 ítems, en donde se observa de forma general que la mayoría de las mujeres que dieron respuesta al instrumento indican facilidad para la resolución de problemas y para el aprendizaje de las asignaturas relacionadas. A continuación se presentan las gráficas con los porcentajes obtenidos:

"Me resulta sencillo aprender y resolver problemas matemáticos".

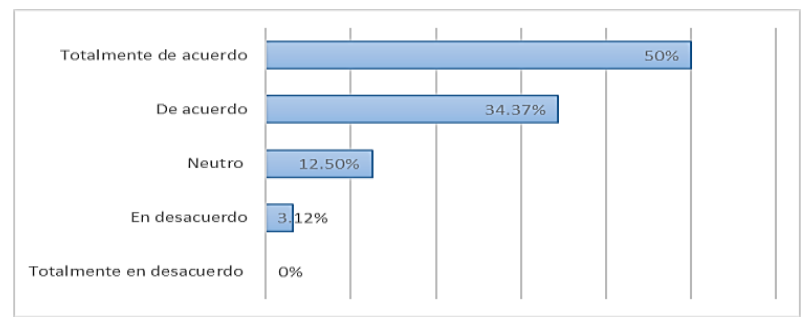

Gráfico 4 Facilidad para matemáticas

Fuente: Elaboración propia

"Me resulta sencillo aprender y resolver problemas del área de las ciencias".

Gráfico 5 Facilidda en ciencias

Fuente: Elaboración propia

c. Los siguientes ítems se refieren al apoyo social, docente y familiar. El bloque consta de 5 ítems, en las respuestas se observa que consideran las encuestadas que sus profesores las motivaron hacia estudio en áreas STEM, en relación con los padres, se observa que la madre proporciona un mayor apoyo con respecto al padre. A continuación se presentan las gráficas con los porcentajes obtenidos:

"Durante mi educación básica tuve docentes que me motivaron a estudiar asignaturas STEM (Geometría, Aritmética, Biología, Química, etc.)”.

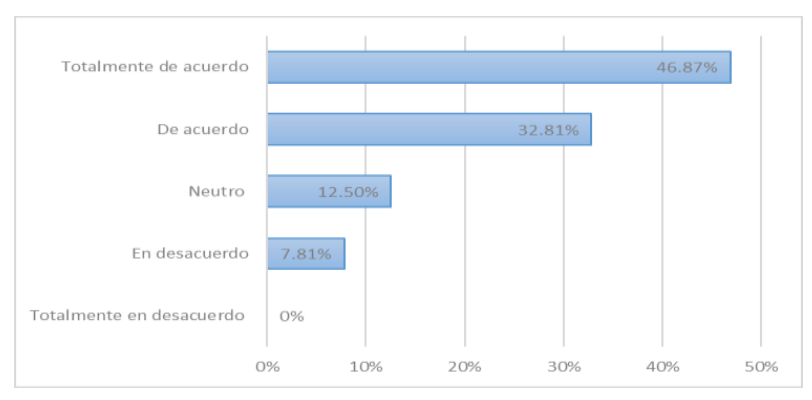

Gráfico 6 Motivación en STEM

Fuente: Elaboración propia

"Mi familia fue importante en mi proceso escolar".

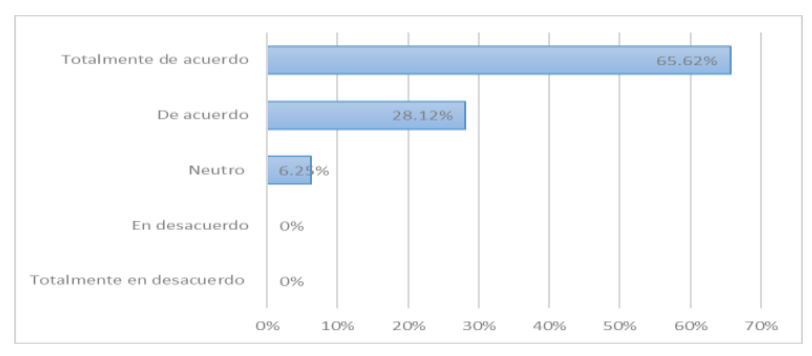

Gráfico 7 Familia

Fuente: Elaboración propia

"Mi madre me motivó y apoyó en mi elección de carrera". 


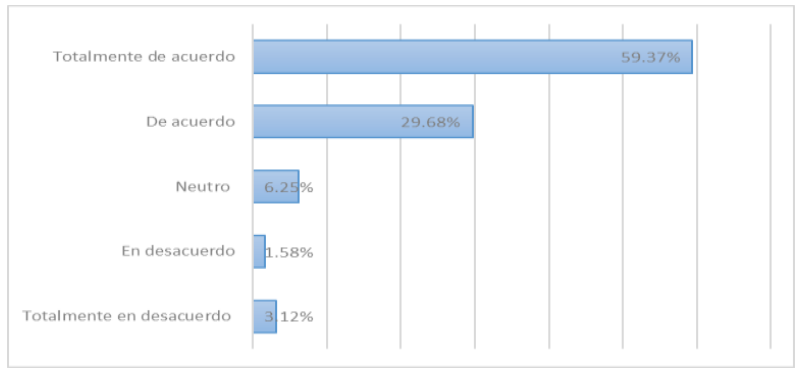

Gráfico 8 Apoyo materno

Fuente: Elaboración propia

"Mi padre me motivó y apoyó en mi elección de carrera".

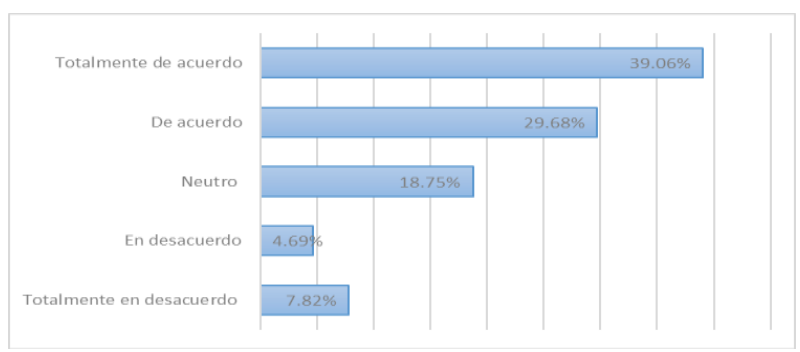

Gráfico 9 Apoyo paterno

Fuente: Elaboración propia

"Considero que las creencias que tienen las madres y padres con respecto a las habilidades matemáticas o científicas de sus hijas impactarán en la forma en la que ellas evaluarán sus propias capacidades".

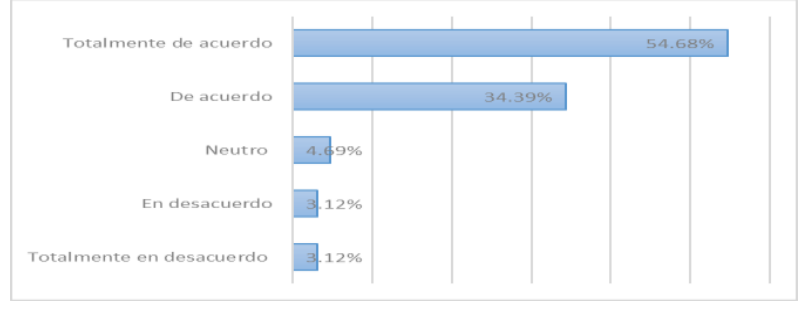

Gráfico 10 Creencias familiares

Fuente: Elaboración propia

d. Estudiar una carrera es la parte inicial del camino, lo siguiente es el trabajo y el desarrollo profesional, es por ello por lo que se indagó sobre la satisfacción en el trabajo y el clima laboral existente.

El bloque consta de 4 ítems, en las respuestas se observa satisfacción en general de las mujeres, con respecto a su elección y satisfacción en el trabajo, además de considerarlo satisfactorio y agradable. A continuación se presentan las gráficas con los porcentajes obtenidos:
"No tuve dificultades para obtener trabajo en el área de mi especialidad".

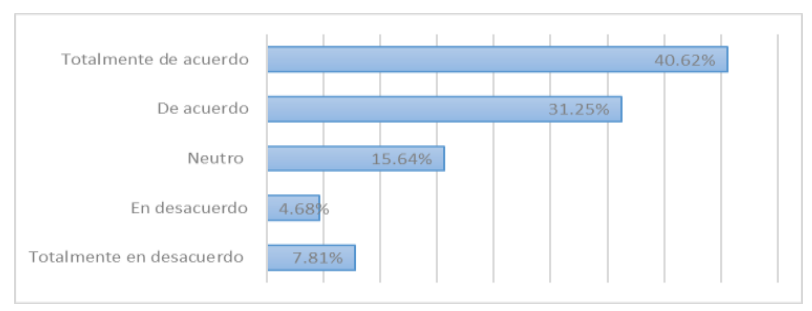

Gráfico 11 Dificultad laboral

Fuente: Elaboración propia

"Me siento satisfecha con mi trabajo".

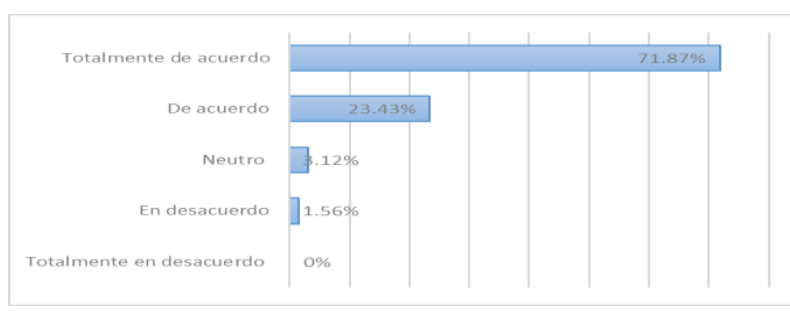

Gráfico 12 Satisfacción en el trabajo

Fuente: Elaboración propia

"Me gusta mi trabajo actual, lo encuentro retador".

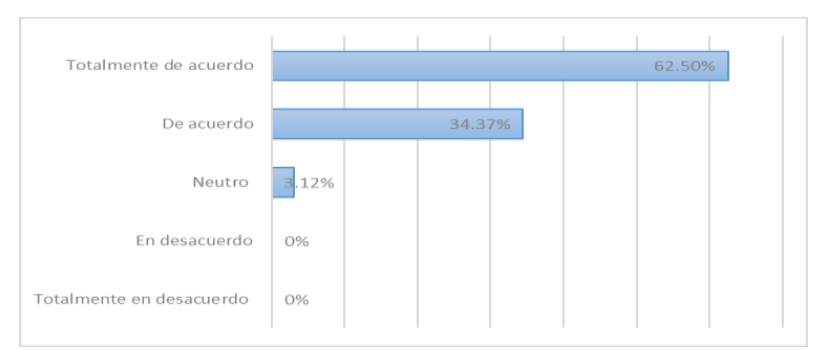

Gráfico 13 Trabajo reatdor

Fuente: Elaboración propia

"El ambiente donde trabajo actualmente es agradable".

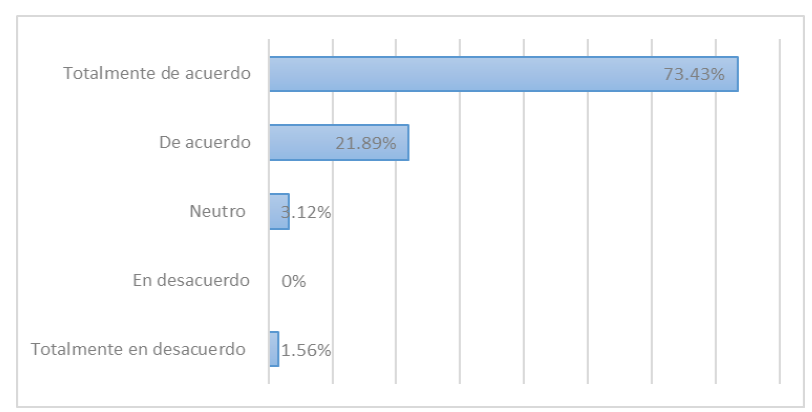

Gráfico 14 Ambiente laboral

Fuente: Elaboración propia 
e. En relación con el bloque que analiza los estereotipos de género, consta de 3 ítems, se observa que las encuestadas perciben que los hombres muestran un mayor interés que las mujeres en las áreas STEM, sin embargo, no se identifica una diferencia en sus habilidades; también se indica que en cuestión a trato entre género, durante sus años de estudiantes percibieron una diferencia en el trato hacia las mujeres y a los hombres. A continuación se presentan las gráficas con los porcentajes obtenidos:

"Los hombres y las mujeres tienen las mismas habilidades matemáticas y cientificas en general".

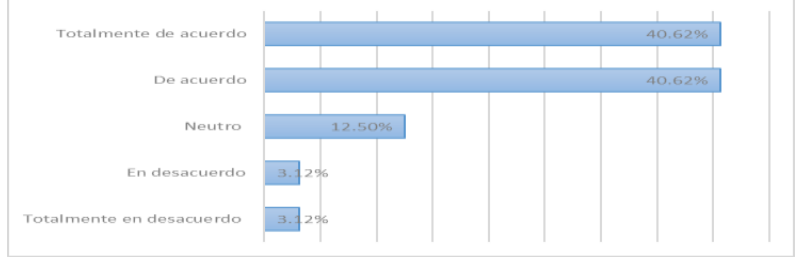

Gráfico 15 Habilidades por género

Fuente: Elaboración propia

"Durante mis años de estudiante percibí un trato diferenciado a hombres y a mujeres".

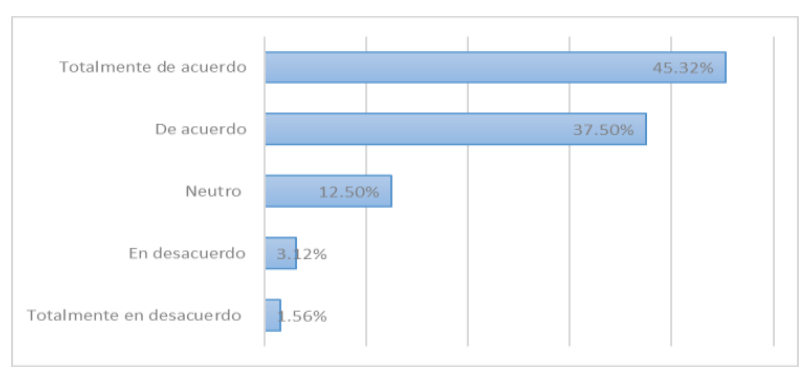

Gráfico 16 Trato diferenciado

Fuente: Elaboración propia

"Considero que las mujeres y los hombres tienen distintas habilidades matemáticas".

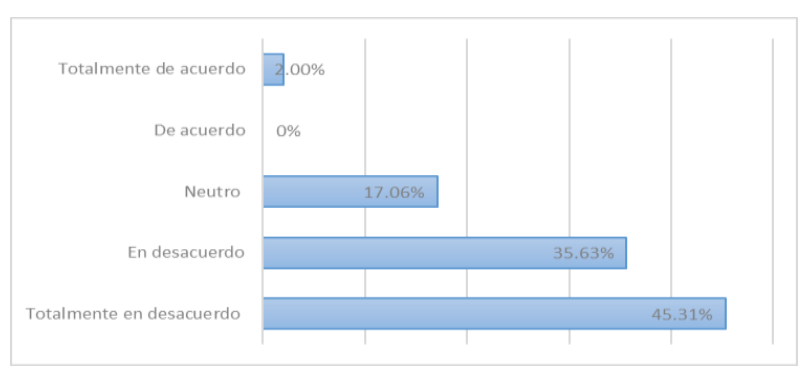

Gráfico 17 Habilidades por género

Fuente: Elaboración propia f. En el análisis de las habilidades o competencias, las encuestadas consideran que las competencias más empleadas son las siguientes (habilidades duras): razonamiento analítico, administración de proyectos, análisis de bases de datos; (habilidades blandas): capacidad de adaptación, actitud positiva, trabajo en equipo, y la expresión oral y escrita. A continuación se presentan las gráficas con los porcentajes obtenidos:

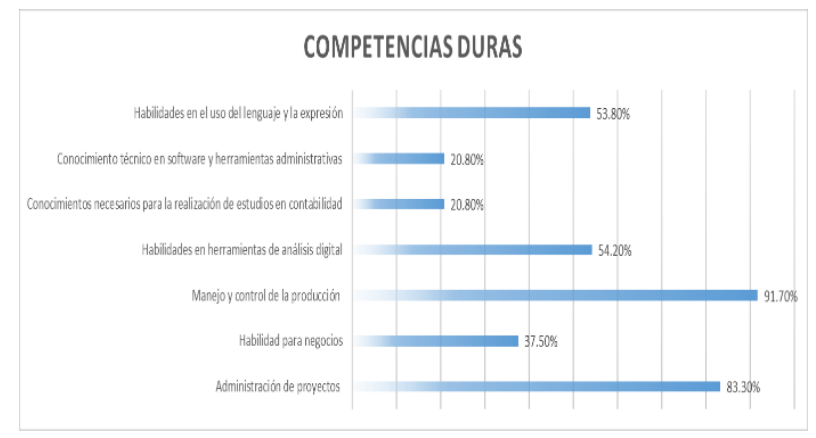

Gráfico 18 Competenicas duras

Fuente: Elaboración propia

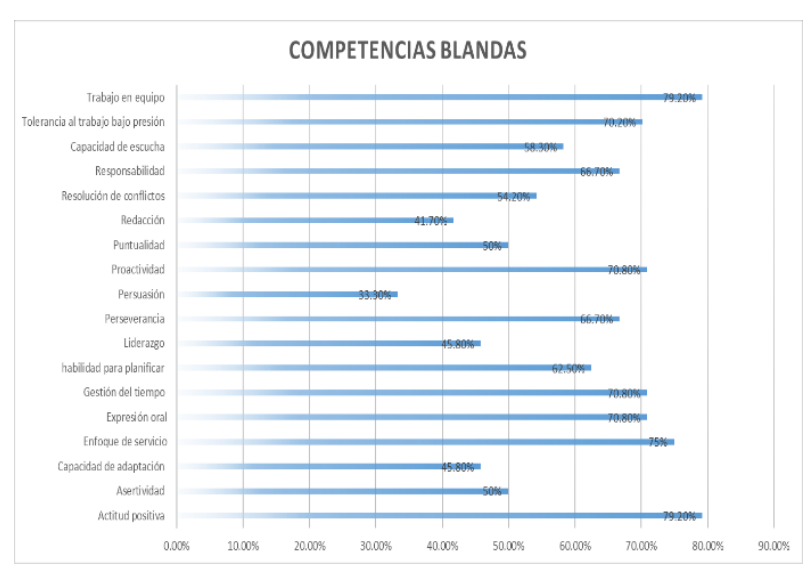

Gráfico 19 Competenciaas blandas

Fuente: Elaboración propia

\section{Conclusiones}

Las respuestas que se dieron a las preguntas, y la posterior revisión de la literatura, permiten visualizar que las razones de la diferencia en el desempeño matemático en niñas y niños, así como la brecha entre hombres y mujeres que estudian una carrera en las áreas STEM es multifactorial.

Conocer de primera mano la percepción de mujeres que tuvieron la experiencia de estudiar y trabajar en una especialidad del STEM fue muy enriquecedor, ya que son mujeres que se destacan profesionalmente en ámbitos académicos, científicos, técnicos o tecnológicos. 
Los resultados muestran que se perciben diferencias de trato entre hombres y mujeres durante la edad escolar, también que las creencias de los padres y las madres con respecto a las habilidades matemáticas y científicas de sus hijas impacta en su autoconfianza.

Es importante, para combatir la brecha y los estereotipos de género, el apoyo de los docentes dentro del aula y el de la familia, así como su respaldo hacia la carrera que han decidido cursar, tratar de eliminar desde lo que social y culturalmente identifica a las mujeres, como son las carreras con el cuidado, la salud y la cosmética; se requiere fortalecer los modelos de mujeres en áreas STEM, tal como se realiza con la iniciativa "NIÑASTEM PUEDEN", impulsada por la Secretaría de Educación Pública en colaboración con la OCDE, con el propósito de coadyuvar en conjunto con profesionistas mexicanas destacadas, para motivar a las niñas, adolescentes y jóvenes a participar en el área científica, tecnológica e innovación a través de una variedad de actividades de difusión y divulgación;

El proyecto en mención tiene por objetivo: "promover en niñas y adolescentes el convencimiento de que son capaces de emprender carreras exitosas en Ciencias, Tecnología, Ingenierías y Matemáticas con independencia de su condición de género, empoderándolas a reconocer y usar sus conocimientos y habilidades". (SEP, 2019), a fin de garantizar que las niñas y jóvenes adquieran las mismas herramientas y habilidades que los hombres, y despertar su interés y amor por la ciencia. Los principales mensajes que el proyecto emite son entre otros, los que a continuación se mencionan:

- Las niñas mexicanas son capaces de aprender a aprender en ciencias, tecnología, ingeniería y matemáticas.

Las niñas pueden optar por estudiar carreras de ciencias, tecnología ingeniería y matemáticas para comprender su entorno y construir un plan de vida plena.

- Las niñas que optan por una carrera STEM son capaces de conciliar su vida personal y familiar con la laboral.
Otro factor relevante para el éxito es la resiliencia personal, como se revisó, es menor el porcentaje de mujeres que hombres que estudian carreras en áreas STEM, sin embargo, la tasa de titulación es mayor en ellas (UNESCO, 2015; citado por Zuleta \& Guil, 2018); la persistencia $\mathrm{y}$ actitud prevalece en su trayectoria profesional.

En lo que se refiere al aspecto laboral dentro de las carreras STEM, se tiene la creencia que existe un ambiente adverso hacia las mujeres, por ello fue alentador conocer las respuestas con respecto a esta variable, ya que a la mayoría de las encuestadas les fue fácil obtener un empleo en el ámbito de su especialidad, encuentran su trabajo retador y perciben un ambiente laboral agradable.

Otro de los hallazgos interesantes fue en la selección de habilidades y competencias duras y blandas, porque permite visualizar las que son las necesarias en el mercado laboral actual, y que las profesionales STEM de la muestra han desarrollado para sobresalir o mantenerse.

Este estudio permitió identificar áreas de interés para posteriores estudios, como es el indagar como fue el proceso de aquellas mujeres que consideran que no tienen una facilidad para el estudio o para la aplicación de las matemáticas, y que sin embargo, están trabajado en área STEM; o a quienes su familia no apoyó en su elección de carrera, conocer la respuesta a la preguntas: ¿cuáles fueron sus motivaciones y reforzadores para continuar con su meta?, sin dejar de lado el indagar las estrategias que emplean para lograr que la combinación de su carrera y trabajo, con un rol socialmente asignado a la mujer como esposa y/o madre se realice con éxito.

\section{Discusión}

La educación STEM ha cobrado una relevancia en los últimos años, lo cual significa que existe una atención particular a la educación, formación y capacitación en estas áreas del conocimiento y desarrollo. Las tendencias pedagógicas y didácticas se orientan a prácticas metodológicas utilizando técnicas que permitan adquirir un aprendizaje significativo, como son los basados en proyectos, el aprendizaje situado, la clase invertida, el rediseño del aula y las capacidades digitales, por mencionar sólo algunos recursos que robustecen el modelo por competencias. 
Por otra parte, surgen diversas iniciativas para impulsar la participación de las niñas en carreras STEM, entre las que se encuentran las siguientes opciones:

Mentorías: Elegir una carrera técnicouniversitaria exige información previa. Las jóvenes necesitan referentes que las ayuden a tomar la mejor decisión y las alienten a desarrollar sus habilidades cognitivas $\mathrm{y}$ socioemocionales.

Talleres focalizados: Asociaciones $\mathrm{y}$ empresas promotoras del modelo STEM que convocan a la sociedad a apoyar la creciente participación de niñas y jóvenes en actividades científicas.

Campañas de promoción del modelo STEM desde el hogar: Permite orientar a los padres de familia a evitar los estereotipos de género y promover un ambiente de interés por la ciencia y la tecnología, sin distinción de género. El elemento crucial será la detección temprana de las habilidades de las niñas y jóvenes.

Políticas públicas incluyentes: Las autoridades educativas tienen el papel de difundir la ciencia y la tecnología en los ámbitos académico, social y económico; la iniciativa de la Secretaría de Educación Pública (SEP): Niñas STEM, es el primer paso para alcanzar el objetivo de promoción en las niñas y adolescentes cursar carreras exitosas en ciencias, tecnología, ingenierías, y matemáticas.

\section{Referencias}

Arredondo-Trapero, F.G., Vázquez-Parra, J. C., \& Velázquez-Sánchez, L. M. (2019). STEM y brecha de género en Latinoamérica. Revista de El Colegio de San Luis, 9(18), 137-158. https://doi.org/10.21696/rcs19182019947

Basco, A.I., Lavena, C (2019). Un potencial con barreras: la participación de las mujeres en el área de ciencia y tecnología en Argentina. Instituto para la Integración de América Latina y el Caribe (INTAL) Sector de Integración y Comercio. Washington, DC: BID https://publications.iadb.org/publications/spanis h/document/Un_potencial_con_barreras_la_par ticipaci\%C3\%B3n_de_las_mujeres_en_el_\%C 3\%A1rea_de_Ciencia_y_Tecnolog\%C3\%ADa en_Argentina_es_es.pdf
Canché, Farfán \& Simón (2011). Género y talento en matemáticas. Memoria de la XIV Escuela de Invierno en Matemática Educativa. CINVESTAV. Pp. 287-291.

Del Río, M., Strasser, K. \& Superreguy, M. (2016). ¿Son las habilidades matemáticas un asunto de género? Los estereotipos de género acerca de las matemáticas en niños y niñas de kinder, sus familias y educadoras. Calidad en la educación.

https://scielo.conicyt.cl/scielo.php?script=sci_ar ttext\&pid=S0718-45652016000200002

Givord, P. (2020), ¿Los niños y las niñas tienen actitudes similares hacia la competencia y el fracaso? PISA in Focus, No. 105, OECD Publishing, París. https://www.oecdilibrary.org/education/do-boys-and-girls-havesimilar-attitudes-towards-competition-andfailure_a8898906-en

Inga, S. M., Tristán, O. M. (2020). ¿Por qué hay pocas mujeres científicas? Una revisión de literatura sobre la brecha de género en carreras STEM. aDResearch: Revista Internacional de Investigación en Comunicación, (22), 118-133. https://dialnet.unirioja.es/servlet/articulo?codig $\mathrm{o}=7302725$

López-Bassols, V., Grazzi, M., Guillard, C. y Salazar, M. (2018). Las brechas de género en ciencia, tecnología e innovación en América Latina y el Caribe: resultados de una recolección piloto y propuesta metodológica para la medición. Washington, DC: BID. https://publications.iadb.org/es/las-brechas-degenero-en-ciencia-tecnologia-e-innovacion-enamerica-latina-y-el-caribe-resultados

Organización para la Cooperación y el Desarrollo Económicos (2016). Innovating Education and Educating for Innovation: The Power of Digital Technology and Skills. París: OECD Publishing. https://www.oecd.org/fr/innovation/innovatingeducation-and-educating-for-innovation9789264265097-en.htm

Organización para la Cooperación y el Desarrollo Económicos (2017). Panorama de la educación. OECD Education at a lance. París: OECD Publishing.

https://www.oecd.org/education/skills-beyondschool/EAG2017CN-Mexico-Spanish.pdf 
Secretaría de Educación Pública (2017). Niñas

STEM pueden (en línea).

http://ninastem.aprende.sep.gob.mx/en/demo/ho me_

Zuleta, A., \& Guil, A. (2018). Las mujeres en las ingenierías y las "ciencias duras". Investigación y género. Reflexiones desde la investigación para avanzar en igualdad: VII Congreso Universitario Internacional Investigación y Género. SIEMUS (Seminario Interdisciplinar de Estudios de las Mujeres de la Universidad de Sevilla). pp 865-871

Arredondo-Trapero, F.G., Vázquez-Parra, J.C., \& Velázquez-Sánchez, L. M. (2019). STEM y brecha de género en Latinoamérica. Revista de El Colegio de San Luis, 9(18), 137158. https://doi.org/10.21696/rcs19182019947. 